\title{
Pre-analysis plan for: Wins and losses in collective actions: Evidence from the field *
}

\author{
Biljana Meiske ${ }^{\dagger} \quad$ Raisa Sherif $^{\ddagger}$
}

June 11, 2021

*We thank Jacob Roshan, Andrea F.M. Martinangeli, Anik Ashraf, Sven Simon. The ethics clearance for this project is obtained from the ethics council of University of Munich with id Project 2021-04. We are grateful for the financial support from the Max Planck Society.

${ }^{\dagger}$ Max Planck Institute for Tax Law and Public Finance, Munich biljana.meiske@tax.mpg.de

${ }_{\ddagger}$ Max Planck Institute for Tax Law and Public Finance, Munich raisa.sherif@tax.mpg.de 


\section{Introduction}

From climate change and preservation of biodiversity, to ensuring political liberties and equality rights, many social problems of interest can be represented as issues that, at best would be resolved on the global level, but that also have their representations on a smaller scale. For example, signing a petition requesting stricter regulation to stop deforestation on the EU level, or joining a local initiative to protest against the planned use of the forestland for surface mining - both constitute an effort toward resolving the issue of deforestation, but on different scales. Alternatively, approaching the problem on a smaller scale could also take the form of selecting a single dimension of the given problem and focusing efforts on resolving that one dimension (as for example requesting same-sex marriage rights, as one step towards complete equality of rights).

In his seminal article Small wins: Redefining the scale of social problems $(1984)^{1}$, a psychology scholar Karl Weick presented the case for breaking down large "social problems" into smaller and more manageable parts. The main claim of the article is that, while humans need to perceive issues as problems in order to engage in activities that remedy them, depicting social problems as large and complex leads to feelings of hopelessness, which in turn reduce the probability of taking actions to solve them. Instead, he proposes recasting large social problems as a line of smaller issues, each of which when solved leads to a small win - "a concrete, complete, implemented outcome of moderate importance" (p. 40). He theorizes that achieving one small win sets in motion factors that increase the likelihood of achieving another one, and in turn leads to a consistent line of development towards the aimed target.

While Weick looks at how one small win facilitates the next one, in this work we study the effect that achieving a small win (or failing to do so) has on the willingness to contribute towards achieving a related, but larger target. Considering that efforts to resolve social problems often take the form of protesting, signing petitions, voting,

\footnotetext{
${ }^{1}$ Karl E Weick. Small wins: Redefining the scale of social problems. American Psychologist, 39(1): 40, 1984.
} 
changing consumption patterns etc., which all constitute some form of a voluntary contribution mechanism, we focus on the framework in which individuals participate in public good games of different scales. To test the effect of small wins, we administer a field experiment in which subjects first participate in a single round of threshold public goods game (TPGG) in a small group, and then proceed to play a similar TPGG, but in a larger group. Similar to the case where a certain number of signatures on a petition are necessary to trigger some consequences, in the first phase, the threshold serves to distinguish successfully achieving a small win from failing to do so. This in turn allows us to study the effect that having achieved a small win, or equivalently having suffered a small loss, has on the willingness to contribute in the subsequent bigger scale collective action.

\section{Design}

\subsection{Experimental setting}

The experiment is planned in the backdrop of an online course on environment and waste management. Due to Covid19, school education in the state of Kerala moved to a digital format, with online teaching. Students in selected schools are given access to an online learning module on environment that contains short video lectures and interactive games, as part of their curriculum. The lectures cover issues regarding the need to preserve the environment and how to adequately manage waste. The course takes on average 6 hours to complete. However, the students do not do the course in one sitting. On average students spend 30 - 45 minutes on the course a week; thus taking up to 8 - 12 weeks to complete the entire course.

Once the subjects complete the course, they are divided into groups of three for the first stage, which we will refer to as "small threshold public goods game". Subjects are randomly matched into groups of three and assigned to a task of making bags from old newspaper that can replace single-use plastic bags. Subjects are given access to short training videos on how to make these bags and are given a week to 
finish the task. They are informed that, if they as a group make 30 of these bags, all three members would win a voucher of 100 INR each. If the group fails to meet this threshold, no member receives the voucher. The number of bags a subject makes is the individual contribution to the threshold public goods game and there is no limit on this individual contribution. The rules are public information. Additionally, the subject does not know the identity of the other members in the group and cannot communicate or co-ordinate with them on the task. Once the task is completed, subjects are informed whether their group met the threshold or not, and the members of the winning groups receive the vouchers. By this stage subjects are aware about their own contributions and whether or not their group met the threshold, but not the contributions of other members in the group.

The subjects then move to the second stage, which is the large threshold public goods game. Here the subjects play in groups of 50. They are randomly matched with 49 other students. The threshold to win is sufficiently adjusted to reflect the size of the new groups to 500 bags. The members of the groups that meet this threshold are given vouchers of value 100 INR each.

\subsection{Data collected}

The following data is collected for the study.

1. Contributions to small and large threshold public goods and data on wins and losses.

2. Attitudes towards pro-environment activities and awareness levels of environment issues among the students. This is collected both at the start and end of the course and used to control for intrinsic motivation and awareness levels.

3. Demographics including age, gender, and income. 


\section{Empirical Strategy}

Denote the contribution to the small threshold public good (first phase of the experiment) of individual $i$ by $C_{S}^{i}$, and the contribution to the large threshold public good (second phase of the experiment) by $C_{L}^{i}$. Moreover, let $I_{w i n}^{i}$ be a dummy variable that equals 1 if the sum of contributions in the group in which individual $i$ played the small threshold public good game was equal or larger than the set threshold (and thus the local public good provision was achieved). In order to test the effect of achieving the small public good, relative to failing to do so we run the following OLS estimation:

$$
C_{L}^{i}=\alpha+\beta C_{S}^{i}+\gamma I_{w i n}^{i}+D^{i}+X^{i}+\epsilon^{i}
$$

where $D^{i}$ denotes a battery of demographic variables (age, gender, income) and $X^{i}$ stands for an index capturing intrinsic motivation regarding pro-environmental behavior, as measured at the end of the course (thus prior to the participation in the first public good game).

Furthermore, in order to test whether achieving the threshold in the small public good game has a different effect on the free-riders relative to the contributors we run the following OLS estimation:

$$
C_{L}^{i}=\alpha+\beta C_{S}^{i}+\gamma I_{w i n}^{i}+\delta I_{w i n}^{i} * I_{\text {free-rider }}^{i}+D^{i}+X^{i}+\epsilon^{i}
$$

where $I_{\text {free-rider }}^{i}$ is a dummy variable that equals 1 if the participant $i$ contributed zero in the small public good game.

Whereas the previous estimation allows us to study whether contributors (pulled into single group) and free-riders react differently to having successfully achieved the small public good, we also analyze whether the level of previous contribution influences the sign and size of the effect.

$$
C_{L}^{i}=\alpha+\beta C_{S}^{i}+\gamma I_{w i n}^{i}+\epsilon I_{w i n}^{i} * C_{L}^{i}+D^{i}+X^{i}+\epsilon^{i}
$$

